REDES- Revista hispana para el análisis de redes sociales

Vol.11,\#3, Diciembre 2006

http: // revista-redes.rediris.es

\title{
Redes socio-técnicas y participación ciudadana: propuestas conceptuales y analíticas para el uso de las TI Cs
}

\author{
Samira Kauchakje (Pontifícia Universidade Católica do Paraná - PUCPR); Manoel \\ Camillo Penna (PUCPR); Klaus Frey (PUCPR) y Fábio Duarte (PUCPR) ${ }^{12}$
}

\section{Resumen}

La sociedad contemporánea tiene en los flujos de la información una de sus características más destacadas, siendo las nuevas tecnologías de la información y comunicación uno de sus pilares. En este contexto, el paradigma de las redes adquiere fuerza, sea cualitativamente (sociedad en red), o sea como instrumento analítico. Este último es el punto que desarrollaremos en el artículo desde un punto de vista conceptual y metodológico, para acercanos a estudios de caso desarrollados sobre los principios de las redes sócio-técnicas y la utilización de las TICS.

Palabras clave: Análisis de Redes Sociales - Redes socio-técnicas - Tecnologías de Información y Comunicación - Participación ciudadana.

\begin{abstract}
Contemporary society has the flows of information as one of it main characteristics and the information and communition technologies (ICTs) as one of its axes. In this context, the paradigm of networks has a special relevance, both for it qualities (the network society is a powerful metaphor) and as an analytical tool. It is the point we develop in this article, from a conceptual and methodological point of view, from what we discuss some study cases our group has develop on the use of ICTs and the construction of socio-technical networks.
\end{abstract}

Key words: Social network analysis - socio-technical networks - Information and Communication Technologies - democratic participation.

\section{I ntroducción}

La sociedad contemporánea tiene en los flujos de la información una de sus características más destacadas, siendo las nuevas tecnologías de la información y comunicación uno de sus pilares. En este contexto, el paradigma de las redes adquiere fuerza, sea cualitativamente (sociedad en red), o sea como instrumento analítico -este es el punto que desarrollaremos en el artículo. En las ciencias sociales o ingenierías, las propiedades de las redes vienen siendo cada vez más usadas en la identificación y análisis de fenómenos cuyas cualidades parecen ser mejor percibidas y explicadas con tal instrumental conceptual y metodológico.

\footnotetext{
1 Enviar correspondencia a: Samira Kauchakje (samira.kauchakje@pucpr.br); Manoel Camillo Penna (penna@ppgia.pucpr.br); Klaus Frey (klaus.frey@pucpr.br); Fábio Duarte (duarte.fabio@pucpr.br).

${ }^{2}$ Este artículo está basado en la investigación internacional "Redes técno-sociais e gestão democrática da cidade", coordinada por la Profa. Tamara Egler (IPPUR/UFRJ), y el apoyo financero del Fondo Regional para la Inovación Digital en Latino America y el Caribe (FRIDA) de LACNIC; y en la
} 
En relación a la gestión territorial (considerando aspectos urbanos, ambientales o sociales) y substituyendo la visión jerárquica de la gestión, en donde el planeamiento y las decisiones provenían de órganos generalmente ligados al ejecutivo, nuevos arreglos institucionales y de cooperación se están estableciendo en forma de redes. En esta estructura, los puntos no se relacionan por subordinación y sí por afinidades - algunas sociales, otras culturales, económicas o funcionales. Parte de esas redes tienen su origen o son potenciadas por las tecnologías de la información y la comunicación. Son llamadas redes socio-técnicas, porque son redes que envuelven la constitución de una organización entre agentes sociales estimulada y mediada por instrumentos tecnológicos y lenguaje codificado para que los lazos de relación entre emisores y receptores (nodos) se hagan efectivos. En este sentido es fundamental la comprensión de las relaciones sociales, de los instrumentos tecnológicos y de la base territorial como elementos interdependientes de la constitución de las redes socio-técnicas y, por esta razón, son necesarias investigaciones interdisciplinares complementarias.

Envueltos en investigaciones en diversas escalas y temáticas, nuestra intención en este artículo es en un primer momento consolidar conceptualmente el término de redes socio-técnicas de forma interdisciplinar. A continuación procuramos analizar los aspectos metodológicos del análisis de las redes socio-técnicas, rescatando el histórico del análisis de las redes sociales para llegar a las especificidades del análisis de redes que envuelven tecnologías de la información y comunicación contemporáneas. Finalizamos presentando brevemente algunos casos en los que venimos trabajando el análisis de las redes socio-técnicas, explorando diferentes escalas y temáticas.

\section{Las redes y las redes socio-técnicas}

Las redes están formadas por entidades y por relaciones entre esas entidades; una relación es un conjunto de lazos de relacionamiento entre entidades, y las entidades que poseen un número de lazos de relacionamiento mayor a 1 son llamadas nodos. Las entidades y las relaciones apenas forman redes cuando posibilitan la existencia de o son demandadas por el otro; o sea, un elemento no puede ser considerado un nodo a no ser que haya articulaciones con otros nodos; y él deja de serlo cuando esas articulaciones se acaban. Son características de las 
redes la agilidad y la flexibilidad para ligar (y desligar) puntos y acciones distantes (Parrochia, 1993), lo que les da un inconstancia latente.

Para Pierre Musso (2004) y Milton Santos (2002) la polisemia de la noción de red sería tanto la causa de su éxito como de su descrédito como base conceptual, llevando a imprecisiones y ambigüedades. En su argumentación filosófica, donde coloca a la red entre la estabilidad organizativa del árbol y el carácter caótico y efímero del humo, Musso (2004: 31) la define como "una estructura de interconexión inestable, compuesta por elementos de interacción, y cuya variabilidad obedece a alguna regla de funcionamiento".

Las redes no son, por tanto, apenas otra forma de estructura, sino casi una no estructura, en el sentido de que parte de su fuerza está en la habilidad de hacerse o deshacerse de tiempo en tiempo.

A pesar de que algunos autores (Musso, 2004; Parrochia, 1993) reforzaron la idea que las redes son formas antiguas de convivencia humana, ellas tomaron "una nueva forma en los tiempos actuales, al transformarse en redes de información, revitalizadas por Internet" (Castells, 2001: 1). En este sentido, Castells atribuye un papel fundamental a las redes de las telecomunicaciones porque ellas imprimen una nueva dinámica social a los sistemas económicos y políticos, proporcionando una nueva "morfología social" de la emergente sociedad en red (Castells, 1999).

Con esto se introduce el problema de la tecnología. La existencia de las redes no depende de las tecnologías de la información - o de cualquier tecnología. La expansión del HIV en el mundo, así como los análisis de mapeamiento del nodo originario y de la dinámica de esa expansión son ejemplos de redes (Rothenberg et al, 1998) que se forman independientemente de la tecnología.

Las redes socio-técnicas envuelven la constitución de una organización entre agentes sociales, estimuladas y mediadas por instrumentos tecnológicos y lenguaje codificado, para que los lazos de relacionamiento entre emisores y receptores (nodos) se hagan efectivos. No se trata de una red social que usa instrumentos tecnológicos tan sólo como soporte. Las redes socio-técnicas tienen como característica que la tecnología estimula, mantiene y amplía su establecimiento. El arte postal de inicios del siglo 20, donde los artistas diseminaban sus trabajos a través del intercambio de correspondencias diseñando sus propios sellos y sobres para escapar de las censuras políticas nacionales, ejemplifica una red socio-técnica 
(Prado, 2003): los artistas son los nodos, y la correspondencia, los lazos de relacionamiento.

En el caso de las redes socio-técnicas del arte postal, una característica importante es que no existía un objetivo más allá de la red; la finalidad de la red del arte postal era su propia dinámica. Pero existen redes socio-técnicas cuyos objetos y objetivos están más allá de la red socio-técnica - están en la sociedad, en el territorio. Es el caso de este artículo, donde discutimos específicamente una posibilidad metodológica del análisis de las redes socio-técnicas basadas en Internet.

Dos suposiciones sirven de base a este trabajo:

(1) La red es un instrumento conceptual y metodológico potente para identificar y analizar fenómenos (o comportamientos de fenómenos) que no se presentarían sin ese instrumental; y

(2) La fuerza del instrumento conceptual y metodológico de la red está en percibir y posibilitar el análisis de fenómenos heterogéneos, o sea, que no puedan ser analizados como pertenecientes a un único sistema.

Esta última suposición es el punto de partida de este artículo: nos interesa identificar y analizar las redes que se forman por la articulación entre las entidades sociales y tecnológicas. No puede haber determinismo en ninguno de los sentidos: los aparatos tecnológicos no son los determinantes supremos de las relaciones sociales, así como sería ingenuo pensar que los aparatos tecnológicos son apenas herramientas de aplicación de las relaciones inherentemente sociales.

En la primera suposición, la red como un instrumental conceptual y metodológico, es útil cuando permite tanto organizar objetos y acciones que no tienen articulación evidente, desvelando algunos fenómenos de difícil percepción, como desestructurar redes cuyas articulaciones son tan claras que tienden a tornarse un sistema ensimismado. Por ejemplo, cuando dejamos de ver un teléfono (o un computador conectado a Internet) como un punto final del sistema de telecomunicaciones para analizarlo como un punto potencial de articulación de actores sociales ligados a una causa, convirtiendo al teléfono en un nodo dentro de una malla comunicativa.

Al analizar las redes de infraestructura tecnológica, Gabriel Dupuy (1985) escribió que deberían ser pensadas en "espacios abstractos, espacios topológicos, espacios de n dimensiones", dejando de verlas como sistemas ensimismados. Aún tomando 
como ejemplo a las telecomunicaciones: coger un teléfono y oír su señal nos indica que el sistema funciona. Sin embargo, por un largo y reciente período en Brasil, la distribución de teléfonos era desigual aún hasta dentro de las ciudades, indicando áreas más o menos privilegiadas económicamente, o políticas selectivas de provisión de infraestructura. Lo mismo aún ocurre con el acceso a Internet: el hecho de existir o no domicilios con acceso a Internet puede ser visto apenas como un indicativo de la dimensión del sistema; sin embargo, al considerar el creciente peso sociocultural de Internet (desde los servicios públicos hasta la participación social), entendemos que ese aparato tecnológico está cada vez más ligado a formas de organización social. Son elementos con propiedades tan dispares (personas y teléfonos) que si pensamos aisladamente, lo máximo que tendremos es el análisis de un sistema ensimismado indicando características genéricas del otro. Sin embargo, si tomamos el concepto de redes, vemos que la heterogeneidad e inestabilidad de las articulaciones son su fuerza motriz - en este sentido, los teléfonos y las personas pasan a ser partes articuladas de una misma red sociotécnica.

Para tomar a la red como un instrumento conceptual y metodológico válido es necesario tener claros 4 puntos diferenciándola de un sistema:

Primero, a diferencia de los sistemas, las redes no necesitan "funcionar" (Duarte, 2002). Segundo, los elementos en un sistema tienden a cumplir funciones determinadas y son substituibles sin perjuicio en la alteración del resultado final; ya que para las redes, cada elemento es único, y su entrada o salida de la red, a pesar de que no perjudique la obtención de determinados resultados, lo transformará cualitativamente. Tercero, las relaciones entre los elementos de un sistema son establecidos antes de su funcionamiento, mientras que en las redes, los elementos se articulan y se desarticulan haciendo que las relaciones se establezcan dinámicamente. Cuarto, en el sistema, si alguna de las relaciones establecidas a priori no ocurren, habrá un "error" o una disfunción que podrá perjudicar el resultado o las relaciones establecidas, mientras que en la red no existe la noción de error, una vez que las articulaciones son inconstantes y mutables.

Una fábrica o un órgano de la administración pública, por ejemplo, a pesar de implicar a herramientas tecnológicas y personas, serían mejor analizados como un sistema. La articulación entre las personas y las herramientas tecnológicas tiene como base un funcionamiento establecido; cualquier funcionario puede ser incondicionalmente substituido sin perjuicio de los aspectos cuantitativos 0 cualitativos del resultado; los funcionarios no tienen características individuales que 
importen al sistema, pero ocupan unas preestablecidas; y en caso de que un funcionario no cumpla su función o la relación preconcebida con otros funcionarios o herramientas tecnológicas, puede existir un error que perjudicará el resultado final.

Se contrapone a tales estructuras jerárquicas tradicionales la actuación de actores sociales a través de redes existentes o la constitución de redes no permanentes motivadas por intereses específicos, con estructura maleable y no jerárquica. En el contexto actual, la organización en red viene siendo empleada por entidades civiles como movimientos ambientalistas, organizaciones no gubernamentales y por acciones políticas globales y locales, éstas con especial interés para ser analizadas como redes urbanas.

En este artículo proponemos contribuir al debate sobre las redes socio-técnicas considerando los siguientes aspectos:

- Las redes socio-técnicas nunca forman totalidades ensimismadas.

- Las redes socio-técnicas adquieren su fuerza en la inconstancia de las articulaciones existentes y posibles.

- Las redes socio-técnicas son al mismo tiempo articuladoras y desestabilizadoras de otras redes y sistemas.

Para poder comprender mejor las características de tales redes socio-técnicas presentaremos en este trabajo abordajes analíticos que nos permitan vislumbrar las interrelaciones e influencias mutuas entre los artefactos técnicos y los procesos dinámicos sociales, enfatizando las formaciones de redes socio-técnicas en el ciberespacio y su relación con las redes sociales convencionales. En una primera parte, serán presentados los aspectos generales de la metodología de análisis de las redes sociales. En secuencia, haremos el abordaje del análisis de las Web para evidenciar las influencias recíprocas entre redes sociales y las estructuras que caracterizan las tramas de hiperenlaces en el ciberespacio - uno de los aspectos innovadores del análisis de redes socio-técnicas basadas en Internet. En la tercera parte, discutiremos específicamente la metodología utilizada en nuestras investigaciones de análisis de las redes socio-técnicas, buscando separar fenómenos cuya percepción y análisis se potencian en la alianza entre abordajes histórico-interpretativos y estructurales estimulado por el mapeamiento de las redes socio-técnicas establecidas en Internet. Concluimos el artículo con algunos posibles campos de aplicación de los abordajes metodológicos propuestos. 


\section{Aspectos metodológicos del análisis de las redes sociales}

El empleo más sistemático del concepto de redes sociales se dio a partir de la década de 1940, teniendo como precursores los trabajos de Radcliffe-Brown y Simmel (Scott, 2000; Wasserman y Faust, 1994; Emirbayer y Goodwin, 1994). Scherer-Warren (2005) identifica dos vertientes en los orígenes del concepto de redes sociales: la primera, iniciada por las ideas de Radcliffe-Brown y sus seguidores a partir de la década de 1940, que procuró en la noción de red una explicación para la estructura social; y la segunda, iniciada por Barnes y seguidores a partir de la década de 1950, que encontró en la noción de red una forma para describir las relaciones sociales primarias de lo cotidiano.

En la historia de la investigación sistemática de más de medio siglo y la prominencia incuestionable del análisis de las redes sociales en los últimos años, la mayoría de los investigadores coincide con que el análisis sociológico a partir del concepto de redes aún no constituye una teoría formal o unitaria en el sentido de especificar leyes distintas, proposiciones, o correlaciones. En lugar de esto, lo que existe es una estrategia amplia para la investigación de la estructura social (Scott, 2000; Wasserman y Faust, 1994; Emirbayer y Goodwin, 1994), apoyándose en el análisis de procesos históricos y en los métodos tradicionales de la etnografía, antropología, de los estudios de caso y de la sociología histórica. Castells (2000) y Graham y Marvin (1996) han evaluado el impacto de la estructura en redes en la reorganización de la sociedad, según los principios de la sociedad de la información; Villasante (2002) y Scherer-Warren (2005), utilizan la perspectiva de redes para entender los movimientos sociales, que unen a individuos y actores colectivos a través de lazos de solidaridad que transcienden a organizaciones empíricamente delimitadas.

Al lado de esos abordajes de cuño interpretativo, preponderantes en los estudios relacionados con las redes en ciencias sociales, interpersonales, de los movimientos sociales y del estado/políticas públicas, implicando cuestiones de temporalidad, espacialidad y sociabilidad, existe una vertiente metodológica estructuralista, que evidencia los procesos interactivos entre individuos a partir de sus relaciones. Aquí la red social es interpretada como una estructura compuesta de elementos en interacción, es decir, un conjunto de actores (nodos) que se interconectan a través de relaciones relativamente estables, no jerárquicas e independientes. Los actores envueltos comparten intereses y son determinados, conjuntamente con las relaciones de la red, por el proceso social que se quiere representar. En este abordaje, la estructura de las relaciones es un concepto central en el análisis de los fenómenos sociales. 
El abordaje estructuralista formó una escuela más homogénea en torno al método de análisis conocido internacionalmente como social network analysis (SNA), al que nos referiremos en adelante con el acrónimo ARS (análisis de las redes sociales). Los métodos y modelos del ARS se desarrollaron a partir de los años 1950, como parte integral de avances en la teoría social, incluyendo la investigación empírica, y teniendo como base a la matemática formal y a la estadística (Wasserman y Faust, 1994). Su desarrollo se dio a partir de tres líneas principales (Scott, 2000): los analistas sociométricos que produjeron avances técnicos a partir de los métodos de la teoría de los grafos; los investigadores de Harvard de los años 1930 que, partiendo de las ideas del antropólogo social británico Radcliffe-Brown, construyeron modelos de relaciones interpersonales y estudios sobre la formación de grupos; y los antropólogos de Manchester, que se apoyaron en desarrollos anteriores para examinar la estructura de relaciones comunitarias en sociedades tribales y de aldea, dando los primeros pasos en la alianza de la matemática con la teoría social.

La consolidación del ARS como metodología se da en los años 1960, con los trabajos de Harrison White, que extendió las investigaciones de la estructura social con base matemática, sintetizando algunos de los discernimientos más importantes propuestos por sus predecesores, y creando una escuela única que fue desarrollada y ampliada por sus seguidores. Otro paso importante para la consolidación del ARS fue la creación de la Red Internacional para el Análisis de las Redes Sociales (INSNA - International Network for Social Network Analysis), por Barry Wellman, en 1978. Se trata de una asociación profesional que congrega a los investigadores interesados en el ARS, que realiza anualmente la International Social Networks Conference, publica las revistas Connections y Social Networks, difundiendo los avances teóricos y técnicos de la investigación (Véase http://www.insna.org).

La identificación de dos abordajes metodológicos principales no implica la creencia en una división dicotómica de los métodos del análisis sociológico. La teoría de la estructuración de Giddens (1989) se fundamenta en los conceptos de estructura, sistema y dualidad de la estructura, pero tiene como dominio básico de estudio a las prácticas sociales ordenadas en el espacio y en el tiempo a través de la acción, que envuelve el poder en el sentido de capacidad transformadora. En esa misma línea, el objetivo del ensayo de Emirbayer y Goodwin (1994) fue el de repensar el análisis de las redes sociales, la sociología teórica, y la sociología histórica a la luz unas de otras, mostrando cómo los trabajos de la vertiente estructuralista estaban enmarcados en modelos interpretativos de relacionamiento entre cultura, agencia y estructura social. En otro ejemplo, Scherer-Warren (2005) relaciona y evalúa 
múltiples paradigmas, técnicas y campos analíticos, sugiriendo un mayor diálogo entre las diversas perspectivas para contribuir al avance científico de este campo del conocimiento. La búsqueda de la conciliación de los abordajes interpretativos y estructuralistas es una de las perspectivas más prometedoras de las metodologías basadas en el análisis de las redes sociales. El abordaje estructuralista permite traer a discusión estructuras e interrelaciones que son de difícil aprehensión por parte de los abordajes interpretativos tradicionales, al mismo tiempo que los resultados del análisis estructural pueden llevar a interpretaciones equivocadas si no fuesen validadas por modelos interpretativos de relacionamientos entre cultura, agencia y estructura social (Frey, Penna y Czajkowski, 2005).

Otra cuestión importante que los métodos de análisis de redes sociales suscitan es la posibilidad de aproximación entre los abordajes de análisis de la macro-sociología y de la micro-sociología. El siglo XX estuvo marcado por la diversidad teórica y metodológica y por un recorrido que fue desde la macro a la micro-sociología. A partir de las bases establecidas por Charles Cooley y William Thomas y de los avances introducidos por Robert Merton, Harold Garfinkel, Vilma Figueiredo (2001) se identifica en la micro-sociología una perspectiva de grande riqueza empírica y de fertilidad de hipótesis. Por su propia naturaleza, los métodos de análisis de las redes sociales permiten que el elemento de estudio pueda ser tanto el individuo con otros entes sociales, tales como la familia, las instituciones, o los grupos sociales, e incluyen necesariamente en sus modelos y abstracciones, a las estructuras que emergen de las relaciones entre ellos. En este sentido, puede cumplir un papel de intermediación entre macro y micro-abordajes de la sociología, entre teorías de estructuras y teorías de acción, entre el imperialismo de supuestas obligaciones colectivas y el relativismo de libertades individuales (Jansen, 2003; Martins, 2004).

Finalmente, cabe aún una breve discusión sobre los abordajes empíricos en las ciencias sociales. La vertiente dominante de las investigaciones empíricas a partir de los años 1940, principalmente en los Estados Unidos, es el abordaje investigativo "cuantitativo" basado en métodos probabilísticos y estadísticos. A partir de esta época, las herramientas estadísticas se consolidaron de modo indiscutible. Previamente las investigaciones sociales empíricas consistían en estudios cualitativos de comunidades, influenciados principalmente por los escritos de Robert Park, William Thomas y George Simmel. Estos teóricos veían los hechos sociales en contextos específicos de tiempo y espacio: los hechos sociales eran considerados como localizados en lugares físicos particulares y en una lógica temporal de uno o más procesos de diversos tipos, como por ejemplo, de sucesión, 
de asimilación, y de conflictos (Emirbayer y Goodwin, 1994). Después de los años 1940, el foco sale de una determinación contextual en dirección a otros tipos de trabajo que tomaban como unidad de análisis no a la comunidad o al grupo social, sino al individuo. Los métodos estadísticos, que tuvieron un avance considerable en este período, contribuyeron decisivamente a este cambio.

El desarrollo de nuevos métodos cuantitativos de análisis, principalmente aquellos basados en datos relacionales, es decir, datos levantados a partir de las relaciones existentes entre los actores en una red, trae una nueva perspectiva de abordaje empírico. Permite que las preocupaciones analíticas se dirijan una vez más a las cuestiones de los campos de interacción y de determinación del contexto, que fueron tan centrales en los sociólogos antes de la revolución de la estadística en los años 1940.

\section{Esfera Web}

Las redes socio-técnicas crecientemente se sirven de las tecnologías de la información y comunicación, entre ellas, de Internet y las tramas de hiperenlaces en la Web para perfeccionar sus formas de cooperación y coordinación social y política. De ahí el abordaje de la 'esfera Web' como referencia analítica para evidenciar las influencias recíprocas entre las redes sociales y las estructuras que caracterizan las tramas de hiperenlaces.

En el modelo emergente de las redes socio-técnicas caracterizadas por el carácter instantáneo de las comunicaciones entre personas y organizaciones la comunicación electrónica altera la geometría de las interacciones sociales, sin la necesidad de contigüidad espacial. Las redes sociales, dinamizadas e incluso constituidas por flujos de información electrónicos, extrapolan el ámbito local para articular, compartir e intercambiar informaciones y recursos a nivel regional, nacional e internacional. Emergen de allí nuevos estándares de relaciones sociales, económicas y culturales entre individuos y organizaciones. Esas articulaciones interescalares contribuyen para la construcción de identidades, tanto para individuos en lo que se refiere a la autoconstrucción social del self (Lamb; Davidson, 2002), como para grupos o movimientos que, en tanto colectivos identitarios y de acción, acaban proyectándose en el espacio de las articulaciones globales (Duarte, 1998) o, como Milton Santos prefiere decir, en los "espacios de la globalización, espacios mundializados reunidos por redes" (2002, p.333).

Los artefactos tecnológicos que dan soporte a las esferas Web temáticas impulsan y moldean a las esferas de identidad y de interacción, proporcionando prácticas de 
colaboración innovadoras; y son las tramas de los hiperenlaces que condicionan y estructuran los caminos potenciales de interacción. Evidentemente, las esferas Web son sólo una de las posibilidades de interacción social en Internet, como son los emails. Pero el carácter más privativo de estos últimos dificulta no sólo el acceso público a tales redes, sino también su análisis ${ }^{3}$.

El desafío de la investigación de las redes socio-técnicas consiste en el análisis conjunto y cruzado, buscando identificar las especificidades, de, por un lado, objetos o artefactos tecnológicos (aquí, específicamente de los hiperenlaces) que tienen incorporadas intencionalidades instrumentales en sus funcionalidades técnicas $y$, por otro, relaciones sociales que, a su vez, son crecientemente condicionadas por la morfología tecnológica. Estamos, por lo tanto, en la búsqueda de un método capaz de captar esta fluidez contemporánea (Santos, 2002: 274), que, siendo básicamente de carácter social, se basa en redes técnicas que condicionan, limitan y, simultáneamente, amplían las posibilidades de interacción social.

Ciertas redes tecnológicas, como las telecomunicaciones, el transporte público o el abastecimiento de agua, obedecen estrictamente a las reglas preestablecidas en general unilateralmente, por empresas públicas o privadas, proveedoras de servicios y tecnologías u órganos reguladores del Estado. Son redes tecnológicas sujetas a un centro conductor. Por otro lado, la red de hiperenlaces, una parte técnica de las redes socio-técnicas, tiene la volatilidad de la dinámica social, expresando intencionalidades de actores locales en la definición de nuevas relaciones sociales apoyadas en otros hiperenlaces, haciendo que sus caminos, dinámicas y extensiones sean maleables sin un único centro inductor.

Así, la naturaleza de las interacciones viabilizadas por la estructura de hiperenlaces puede revelar estrategias de una gestión de identidad institucional, una vez que las organizaciones e instituciones buscan aumentar los efectos de comunicación con relación a sus audiencias organizacionales (Park et al., 2002: 156), modelando a la red de hiperenlaces para dar oportunidad a comunicaciones inter-organizacionales.

\footnotetext{
${ }^{3}$ Se hace particularmente difícil realizar un análisis más amplio de tales redes socio-técnicas, integradas por e-mail lists o por flujos de e-mails, tanto por razones de garantía da preservación de datos personales, como por la necesidad de una profundización cualitativa que sólo acostumbra ser posible con un número limitado de actores; es posible recurrir a entrevistas cualitativas profundas o al método "agenda de tiempo" entre otros métodos de investigación on-line (Neustadt et al., 2002). Se puede tornar bastante interesante la complementación del análisis de las redes socio-técnicas por las esferas Web con tales tipos de análisis cualitativos con los actores más significativos de las redes, conforme discutiremos más adelante.
} 
Aún reconociendo la simultanea condición facilitadora y limitante de la tecnología en sí, la estructura o la "arquitectura" sujeta a una constante transformación de los hiperenlaces representa antes que nada, un proceso de continua apropiación social de la tecnología. Con eso, puede ser comprendida como siendo dinamizada por la vida cotidiana local, por las vivencias y preocupaciones presentes en el día a día de los actores locales, por sus juicios o expectativas en lo que respecta a la posibilidad de contribución de nuevas inter-relaciones globales para sus prácticas cotidianas.

Interpretamos a la arquitectura de los hiperenlaces como expresiones de la búsqueda por la construcción consciente y continua de nuevas configuraciones espaciales, de nuevas identidades, individuales o colectivas, de nuevos espacios de coordinación social, construidos a partir de procesos intencionados de selección, separación y exclusión, representando voluntades y valorizaciones colectivas fundadoras de nuevas relaciones, cuyo análisis, sin embargo, exige abordajes metodológicos innovadores. Entre los principales desafíos Schneider \& Foot (2004) mencionan la cuestión de como lidiar con la naturaleza "co-producida" de la Web, ya que ésta dificulta la atribución de ciertos resultados o realizaciones de agentes específicos en Internet -además de la rapidez y de la imprevisibilidad de la evolución de las estructuras y procesos en Internet.

Como alternativa metodológica para el delineamiento y el mapeamiento de las relaciones entre los sites de la Web se presenta el abordaje del 'análisis de las esferas Web' (Websphere analysis), desarrollada en los trabajos de Foot et al (2002) y Schneider \& Foot (2004). Esta perspectiva posibilita el análisis de relaciones entre productores y usuarios de la red, mediadas por elementos estructurales característicos de los Websites, sobre todo los hiperenlaces, de los cuales es posible inferir la existencia de relaciones de acción informacional o comunicativa. La Web deja de ser vista como una simple colección de sites para ser un conjunto interconectado de recursos digitales dinámicamente definidos que se expanden por múltiples sites, y son considerados relevantes, o relacionados, a un "objeto" o tema central. Según Park et al. (2002: 157), "a través del uso de los hiperenlaces, las organizaciones son capaces de facilitar la comunicación bilateral y la coordinación, pasando y fortaleciendo off-line las fronteras dentro y entre las organizaciones, independientemente de su localización física. Ellas pueden estar interrelacionadas, intercambiar informaciones y mantener relaciones cooperativas a través de hiperenlaces integrados en contextos específicos y en entornos de intereses o proyectos comunes" (p. 157).

El número de hiperenlaces entre dos sites puede ser un indicador del grado de proximidad entre ambos. Los hiperenlaces y los sites son considerados elementos 
simbólicos representantes de las relaciones interinstitucionales en Internet, así como medios de contacto y de intercambio con otras organizaciones o personas en cualquier parte del mundo. Es importante también señalar que las relaciones entre los sites van más allá de los actores que están en los orígenes y en los destinos de los hiperenlaces. Estos mecanismos tecnológicos pasan a constituirse en herramientas para que cualquier ciudadano trace sus rutas de navegación en la Web en función de sus propios intereses. De esta forma, cuanto mejor organizados, más significativos y con un mayor número de hiperenlaces, mayores serán las alternativas disponibles para el acceso a las informaciones.

Apoyados en los estudios emprendidos por Burt (1992), Granovetter (1985) y Mizruchi y Galaskiewicz (1993), Park, Barnett y Yong afirman que las configuraciones de las redes entre organizaciones expresan la calidad y la confianza en las informaciones disponibles, así como el prestigio de los actores centrales en una red. Dentro de una red temática, sea política, de movimientos sociales o de negocios, la evaluación de la credibilidad -y su centralidad en la red- está directamente correlacionada con el número y el origen de los hiperenlaces que a él se direccionan.

El desarrollo de la acción social en las esferas Web ha sido evaluado, según Schneider \& Foot (2004), por tres abordajes distintos. El primero corresponde al análisis discursivo de los sites de la Web, preocupándose esencialmente con su contenido, y no con su estructura. Los contenidos de las páginas son vistos "como inscripciones de prácticas comunicativas por parte de sus patrocinadores y/o usuarios", y métodos de análisis interpretativos son usados para analizar la acción social. La evaluación de la acción a partir de la estructura de los sites es el foco del segundo abordaje. Los estudios en esta línea procuran entender las estructuras de la red formadas en la Web, interpretando esta estructura como una forma particular de prácticas de comunicación. El tercer abordaje, denominado sociocultural por Schneider \& Foot, se basa en métodos de campo, de entrevistas y observaciones de los participantes, en combinación con el análisis de los textos y el análisis estructural.

Considerando la posibilidad de análisis de las relaciones representadas por los hiperenlaces, el abordaje del 'análisis de las esferas Web' puede contribuir para el delineamiento y la aprehensión de las representaciones virtuales de los campos de acción específicos, principalmente aquellos condicionados y modificados sobre la influencia de la esfera Web. 
En esta investigación, la formación y el análisis de una esfera Web ligada a los temas investigados fue una de las etapas iniciales de la metodología. Sin embargo, entendiendo las redes socio-técnicas como aquellas que emergen de la relación entre actores sociales y tecnologías pero cuyas acciones no se restringen a la esfera tecnológica, desarrollamos procedimientos metodológicos específicos, los cuales detallamos a continuación.

\section{Procedimientos metodológicos}

Considerando el debate conceptual y metodológico expuesto, es relevante apuntar los procedimientos generales para la recolección de datos, sistematización, descripción y análisis interpretativo de las redes socio-técnicas que usamos en nuestras investigaciones. Estos procedimientos tienen dos momentos entrelazados, pero con características propias. En un primer momento se procura identificar e interpretar las configuraciones de la red de agentes sociales de la forma en que éstos se presentan en la Web. La reconstitución de esta red se da por la identificación de la arquitectura de los hiperenlaces y de las interconexiones establecidas en los sites de los agentes sociales, así como por las articulaciones existentes entre los agentes sociales, con base en las informaciones disponibles en los sites. En un segundo momento, se emprende el análisis de esta red identificada, buscando aprehender sus aspectos en términos históricos, culturales, económicos y políticos. A pesar de estar relacionados, los dos momentos son desarrollados separadamente, pudiéndo incluso el investigador detenerse en el primero. Sin embargo, llamamos la atención de que los resultados de uno de los momentos ofrecen discernimientos y sugerencias para el otro, en un proceso de sucesivas aproximaciones analíticas interpretativas.

Los procedimientos metodológicos para el análisis de las redes socio-técnicas basadas en las Web adoptadas en nuestras investigaciones fueron:

a) La decisión sobre instrumentos y formatos de recolección de datos.

b) La identificación de los agentes sociales.

c) La tipología de las interrelaciones.

d) El levantamiento de los atributos y la representación gráfica.

d) El análisis.

A continuación, describiremos el elenco de procedimientos metodológicos para la recolección de datos, la formación y el análisis de redes socio-técnicas basadas en la Web. 
a) Decisión inicial sobre instrumentos y formatos de recolección de datos.

La decisión sobre los instrumentos y los formatos de recolección de datos está íntimamente ligada al objeto de investigación, pues es a partir de la reflexión sobre él que el investigador decide cómo iniciar el proceso de identificación, en Internet, de los agentes sociales articulados que componen la red en cuestión. Una estrategia posible es partir de un tema específico, como los derechos humanos, gestión pública o medio ambiente, e iniciar la identificación de sites de agentes sociales envueltos en la temática. Se puede considerar tanto el conjunto total de las diferentes clasificaciones de los agentes sociales - es decir, organizaciones sociales gubernamentales y no gubernamentales, movimientos sociales, instituciones confesionales, personas físicas, entre otros- como también, se puede optar por considerar apenas un conjunto parcial de ellas. Denominamos puertas de entrada a esos actores significativos que componen la primera selección de nodos potenciales de la red socio-técnica estudiada.

La búsqueda inicial por los agentes (puertas de entrada) puede ser hecha tanto a través de un buscador, considerando los más significativos por el orden de presentación del resultado de búsqueda (el cual ya hizo una clasificación por la incidencia), o considerar el conocimiento previo del investigador sobre la temática para la elección de los sites de agentes sociales que califique como significativos en el campo temático. También se puede elegir el site del agente social que el investigador reconozca como relevante o de referencia en el tema/objeto de la investigación, y a partir de los lazos de relacionamiento en él apuntados, seguir la búsqueda por los agentes.

b) Identificación de agentes sociales miembros de la red

A partir de los agentes sociales iniciales (puertas de entrada o agentes significativos) son verificados los hiperenlaces y otros registros descriptivos en los sites para la identificación de los miembros de la red. Esta es una diferencia importante para el análisis de los hiperenlaces en sentido estricto. Mientras ésta considera apenas los hiperenlaces, levantamos en nuestra investigación también otros agentes que, por la explicitación a ellos dada en el site, consideramos significativos -algunas veces, son listados como agentes pertenecientes a la red, pero no poseen sites.

A partir de ese levantamiento en los sites de las puertas de entrada, usamos la técnica Bola de Nieve: para cada información disponible se abre el site del agente citado, y de este se pasa a otros, de forma que se cubra el elenco de relaciones/articulaciones posibilitadas por la aplicación de la técnica. 
Un aspecto fundamental de la técnica bola de nieve es la definición de los criterios de corte que delimitan la muestra. Los criterios de corte son establecidos en dos grandes líneas: la primera, cuando las citas entre los agentes se cierran en sí mismas de forma circular, con el agotamiento de las posibilidades de nuevas aberturas de sites; y la segunda, cuando existe el corte intencionado del investigador a pesar de que hayan otras "citas" entre los agentes. Esta segunda línea de corte se basa en criterios concernientes a la investigación específica, que pueden ser de localidad, tema o clasificación de relaciones.

La intención es que los resultados registrados reflejen la dinámica social, expresando las intencionalidades de los agentes sociales en la construcción y el mantenimiento, en el fortalecimiento y la ampliación de las relaciones sociales sustentadas por el ciberespacio. Esto representaría un proceso de apropiación social de esta tecnología en las prácticas sociales de los agentes, superando, inclusive, barreras relacionadas con la localización geográfica.

c) Tipología de las interrelaciones

Procuramos abandonar la aleatoriedad de listar toda y cualquier interrelación que hubiese en un site y de él proseguir el proceso indefinidamente, para de antemano establecer criterios de corte, tanto para la identificación e incorporación de agentes, como también para la caracterización de interrelaciones que fuesen significativas para la investigación. Eso permitiría que los indicios de base teórico-metodológica escogida para fundamentar las posibilidades interpretativas del análisis, ya estuviesen presentes en la identificación de los agentes y de los lazos de relacionamientos de la red que va a ser constituida.

La tipología de las interrelaciones caracterizada en nuestras investigaciones fue:

- Alianza ideológica: agentes sociales que comparten valores, idearios, concepciones políticas, causas sociales o proyectos societarios, que pueden actuar o no en el mismo campo temático. Sugiere la existencia de lazos fuertes por afinidad, en el sentido de ser miembros "permanentes" de una red a la que se puede acceder para temas, acciones y proyectos específicos y coyunturales. Las afinidades ideológicas, entre tanto, no necesariamente implican la existencia de una práctica de colaboración en proyectos.

- Alianza temática: actuación en el mismo campo temático sin necesariamente compartir valores, idearios, concepciones políticas, causas sociales y/o proyectos societarios. Sugiere lazos más frágiles que, sin embargo, pueden ser activados dependiendo de los pactos de interés y de las oportunidades de acción/relación referidos en la temática. A pesar de no existir lazos fuertes en lo que respecta a las 
afinidades ideológicas, puede haber una colaboración por intereses, lo que muchas veces ocurre en alianzas establecidas entre órganos estatales, empresas privadas y organizaciones no-gubernamentales para la realización de proyectos concretos.

- Alianza en proyecto: cooperación mutua en programas, proyectos, acciones e iniciativas conjuntas. Los socios en el proyecto, al menos durante su desarrollo, son también socios temáticos, pero no necesariamente ideológicos. Sugiere la existencia de lazos entre agentes sociales, que pueden ser fuertes o débiles, permanentes o temporarios, y que se establecen, por lo menos, durante el desarrollo de la acción/proyecto enunciado.

- Apoyo/patrocinio/colaboración: soporte financiero u otro tipo de apoyo (como divulgación), sin representar una implicación directo en la implementación de proyectos. No se puede afirmar que haya afinidad temática y/o ideológica de los apoyos/patrocinadores/colaboradores para con los agentes sociales que están articulados, aunque se pueda inferir que, en algún grado, la afinidad temática es esperada.

El registro de las interrelaciones, a partir de los sites y de acuerdo con esta tipología, puede considerar apenas una de las posibilidades elegidas, o sea, el relacionamiento con un agente social informado en un site será registrado como alianza en proyecto o alianza temática, dependiendo de la inferencia del trazo más fuerte expresado en las informaciones disponibles; pero también puede haber una conjugación entre las clasificaciones, haciendo el registro mixto de varias posibilidades cruzadas, como de una alianza en un proyecto e ideológica, desde que estén coherentes con el objeto y el objetivo de la investigación.

Cabe aún enfatizar que esta clasificación es una construcción del investigador, no siempre pegada a la información disponible en los sites investigados. Puede ocurrir que en un site un agente social sea citado como socio, pero el investigador decida, por las informaciones disponibles, clasificar el relacionamiento como alianza temática, ideológica o como apoyo.

d) Levantamiento de atributos y representación gráfica

Para organizar las informaciones más relevantes se utiliza una ficha de datos formateada para relevar determinados atributos de los agentes sociales. Los ítems de esta ficha dependen del objetivo de la investigación, y son usados para identificar las categorías y atributos de los agentes sociales, permitiendo profundizar el análisis de las interrelaciones. 
Las informaciones organizadas y sistematizadas son entonces convertidas en gráficos por medio del programa NetDraw, que permite representar y visualizar la red identificada, y sirven como base para el análisis. Los recursos del programa (en términos de representación, visualización, formas de lectura y agrupamiento de la red y sus miembros) presentan resultados sugerentes que pueden ser descritos, pero, sobre todo, interpretados y analizados teniendo como base el referencial teórico que da soporte a las hipótesis construidas y a los conceptos que emergerán en el proceso de investigación.

e) Análisis

Los movimientos de identificación, descripción e interpretación son indisociables. Sin embargo, dependiendo del soporte teórico/conceptual, pueden presentar fragilidades en su desarrollo, con un trabajo de identificación interesante, que puede avanzar para una robusta descripción, y desembocar, en una precaria interpretación. Por eso, el análisis exige el conocimiento de los recursos y el método de análisis de la red social (ARS) en sí, y también supone el conocimiento teórico del campo socio-histórico en el cual esta red puede ser aprehendida. Los métodos del ARS utilizan datos relacionales, que procuran levantar informaciones, por ejemplo, sobre el número y los tipos de contactos, lazos y conexiones, que relacionan un agente social con otro. Esas informaciones no pueden ser reducidas a propiedades de los propios agentes individuales, porque las relaciones no son de propiedad de los agentes, pero sí de sistemas de agentes; es decir esas relaciones unen pares de agentes en sistemas de relacionamientos más amplios. Así, en el análisis de la red se procura representaciones formales (matemáticas o algorítmicas) de conceptos de la teoría social, como la estructura social, posición social, centralidad, status y cohesión.

A partir de la aplicación de los métodos del ARS es posible obtener medidas estructurales de la red, que estimulan al investigador en su reflexión sobre el fenómeno, ya que fueron concebidas para representar conceptos de la teoría social.

\section{Redes socio-técnicas en la gestión pública}

Durante nuestra investigación, la discusión conceptual y el refinamiento de los procedimientos metodológicos para el análisis de las redes socio-técnicas, fue acompañada por investigaciones empíricas. Dentro de las redes socio-técnicas ligadas a la gestión pública, destacamos aquellas ligadas a las políticas ambientales, inclusión digital y protección social. 
Ejemplos de cómo las medidas pueden ser usadas en el estudio de las redes sociotécnicas pueden ser encontrados en Penna y Frey (2006). Las medidas pueden ser obtenidas a través de la utilización del software de ARS, por ejemplo, UCINET.

A continuación, presentamos algunos ejemplos de los estudios que vienen siendo desarrollados a partir de los conceptos y procedimientos metodológicos discutidos en este artículo.

\section{a) Red socio-técnica en la gestión urbana}

1. Para la definición y el delineamiento de la temática de red, primero, se decidió que solamente serán registradas aquellas instituciones con actuación explícita en la ciudad de Curitiba, además de las instituciones paranaenses, nacionales o internacionales relevantes para la gestión local en la ciudad, independientemente del área de actuación temática específica de las instituciones. La relevancia estaría caracterizada en los casos, por ejemplo, de que estas instituciones participen en consejos, convenios o proyectos localizados en la ciudad, o en el caso de existir alguna relación específica y explicitada del site con la Prefectura Municipal de Curitiba.

2. Iniciamos el levantamiento a partir del site del Forum Social Mundial, ABONG, Redes RITS y el Portal de la Comunidad del gobierno del Estado del Paraná, que son consideradas las "puertas de entrada" para la identificación de las redes locales de gobernabilidad.

3. Reconocemos a los actores de la red mediante la técnica "Bola de Nieve", o sea, a partir de los actores registrados en los portales de entrada son verificados los hiperenlaces $u$ otros registros descriptivos en los sites para la identificación de los miembros de las redes.

4. Hicimos el registro de las instituciones siguiendo una distinción básica: una alianza en proyectos que caracteriza relaciones entre instituciones en donde los sites evidencian claramente la existencia de acciones o proyectos conjuntos (sugiere la existencia de lazos fuertes (strong ties) entre las instituciones expresadas en proyectos y acciones conjuntas); una alianza temática/ideológica cuya existencia inferimos de la existencia de indicios revelados en los sites por una preocupación compartida en las mismas causas, sin existir indicios para una alianza más directa en proyectos o acciones (sugiere la existencia de lazos débiles (weak ties), los cuales tienen su relevancia al poder ser movilizados si fuese necesario o pertinente); finalmente, los demás links de referencia que apenas son mencionados 
en un campo específico, con algunos atributos relevantes, sin ser más registrados como nuevas instituciones en función de los criterios de corte, lo que también significa que los socios de estos actores no entran más en la identificación de la red. Estos links, sin embargo, pueden ser considerados partes de la policy Websphere conforme a los criterios discutidos anteriormente, al integrar este espacio de intercambio de información y de comunicación viabilizada por la red de hiperenlaces.

5. Finalización de los procedimientos de identificación y recolección de datos de los actores implicados si no hubiera más posibilidades de expandir la búsqueda conforme a los criterios de corte adoptados.

6. Registro integral de los atributos y acciones realizadas por las instituciones conforme han sido previstos en los campos del banco de datos.

7. Producción de los siguientes gráficos a través del software UCINET: "Red de links relacionado con la Gestión socio-técnica del Municipio de Curitiba", "Red de proyectos relacionados con la Gestión socio-técnica del Municipio de Curitiba" y "Red de alianzas relacionadas con la Gestión socio-técnica del Municipio de Curitiba".

8. Análisis de las diversas redes identificadas, teniendo en mente la indagación sobre las posibilidades de que nuevas tecnologías de la información y comunicación sirvan como soporte a las redes de gobernabilidad urbana.

\section{b) Red socio-técnica en la política ambiental}

En virtud de las particularidades del campo de las políticas ambientales, se tornó necesaria una adaptación de los procedimientos. Es necesario resaltar inicialmente el gran número de instituciones que actúan en esta área, una vez que no se adoptó como criterio inicial de corte que las instituciones necesariamente tengan una actuación dirigida a la gestión de las ciudades, es decir, instituciones ambientales con actuación en Curitiba, a pesar de que no tengan ninguna implicación directa en las políticas municipales, fueron incluidas inicialmente en el levantamiento, ya que uno de los intereses de la investigación es verificar la inserción de las redes locales en las redes globales. Con el levantamiento inicial de los movimientos, organizaciones, asociaciones, ONGs y redes ambientales en la ciudad de Curitiba a partir del Site http://www.ambientebrasil.com.br fueron identificadas aproximadamente 50 instituciones. Debido a la imposibilidad, y dentro de las restricciones de tiempo, de levantar todas las instituciones relacionadas con estas 
instituciones iniciales, se optó por adoptar algunas líneas de corte, comenzando por la existencia de una página institucional en Internet, y por la cuestión de que la institución tenga o no su sede en la ciudad de Curitiba; además de eso, fueron desconsideradas todas aquellas instituciones, cuyos sites no dejaron evidentes una implicación clara con las causas ambientales, restando solamente 19 instituciones.

Para el inicio del reconocimiento de las articulaciones mediante la técnica Bola de Nieve, fue iniciada la visita a los Sites de las 19 instituciones catalogadas buscando sus socios en proyectos, socios ideológicos/temáticos o las redes de hiperenlaces conforme al procedimiento estándar adoptado y explicado anteriormente.

En esta identificación fueron contempladas aquellas instituciones con acciones ligadas a temas ambientales; fueron diferenciadas a medida que las acciones eran desarrolladas a nivel local (Curitiba), estatal (Paraná), nacional o internacional, sin embargo, para los tres niveles (estatal, nacional e internacional) se consideraba necesario que las acciones implicasen al municipio de Curitiba.

En virtud de que el número de instituciones era muy grande en el área ambiental, lo que impedía un levantamiento integral en el tiempo disponible, se optó, en este segundo nivel, por considerar apenas aquellas instituciones con acciones ambientales relacionadas al medio urbano, que es el objeto de estudio del proyecto desarrollado por el grupo de investigadores. Adoptando el concepto de policy Websphere analysis, reconocemos que de esta forma no es posible abarcar toda la Websphere de las políticas ambientales, y sí solamente una sub-sphere relacionada con el medio ambiente urbano.

\section{c) Red socio-técnica y la política de inclusión digital y promoción de las TICs}

Las redes de gobernabilidad relacionadas con las políticas tecnológicas, sobre todo las de inclusión digital, fueron levantadas a partir del site del Instituto de Curitiba de Informática $(\mathrm{ICl})$, responsable en Curitiba de los proyectos gubernamentales de inclusión digital en la ciudad. Comparada con la red del área ambiental llama la atención porque se trata de una red mucho más restringida, con menos instituciones del tercer sector implicadas, a la vez que prevalecen instituciones gubernamentales y empresas privadas u ONGs como fundaciones mantenidas por los gobiernos o empresas privadas. Por eso, no se hizo necesario adoptar las mismas restricciones como en el caso de las políticas ambientales, las cuales también son caracterizadas por una mayor diversificación de formas y áreas de actuación. 


\section{d) Red socio-técnica de protección social}

La investigación sobre el conjunto de la protección social en Curitiba (que asegura los derechos sociales como: tierra y habitación, salud, educación, trabajo, seguridad alimenticia y asistencia social), busca aprehender las articulaciones de agentes sociales con mediación de la tecnología de comunicación e información, y, de esa forma, la constitución de una red social socio-técnica de protección social en la ciudad.

La investigación se basó en dos estrategias metodológicas que envuelven, por un lado los procedimientos para el análisis de las redes socio-técnicas basadas en la Web y, también, la recolección de datos por medio de entrevistas.

Por un lado, en los procedimientos basados en la Web, la búsqueda inicial de los agentes (puertas de entrada) fue realizada en un buscador, considerando los más significativos por el orden de presentación del resultado de la búsqueda (lo cual ya hizo una clasificación por incidencia). Aunque, junto a esto, la selección de las puertas de entrada consideró, también, el conocimiento previo del investigador sobre la temática que califica a algunos de los agentes apuntados en el buscador como significativos en el campo temático ${ }^{4}$. A partir de esta selección, los procedimientos siguen lo descrito anteriormente, o sea: la identificación de los agentes sociales miembros de la red; la tipología de las interrelaciones; el levantamiento de atributos y representación gráfica y finalmente el análisis. Cabe resaltar que en el momento de la identificación de los agentes sociales, con la técnica Bola de Nieve, la definición de los criterios de corte que delimitaron la muestra fueron los siguientes: primero, la identificación de los agentes sociales localizados en Curitiba y de sus articulaciones con las citas entre agentes a escala estatal (Paraná) y nacional (Brasil y otros países), hasta el agotamiento de las posibilidades de nuevas aberturas de sites. De esta forma se identifica una red de protección social que tiene como referencia inicial a los agentes de la ciudad. Segundo, el corte intencional del investigador, al incluir a los agentes sociales localizados en Curitiba y sus articulaciones a escala estatal (Paraná) y nacional (Brasil y otros países), los cuales, todavía, necesariamente tienen explicitado en el site algún tipo de actuación o interés en la ciudad. La red identificada en este caso está configurada como de protección social en Curitiba.

\footnotetext{
${ }^{4}$ Para la temática del derecho a la tierra y la habitación, por ejemplo, fueron seleccionadas las siguientes organizaciones como significativas y caracterizadas como "puerta de entrada": ONG "Terra de direito" y COHAB (órgano de la Prefectura responsable por la política habitacional del municipio).
} 
Las entrevistas, por otro lado, son realizadas junto a los agentes sociales significativos en Curitiba (como muestra de conveniencia, no aleatoria). Es importante resaltar que la guía y la técnica del análisis de datos de la entrevista están conjugadas con los procedimientos de la investigación en la Web. Esto es, la guía sigue los mismos ítems y solicita las mismas informaciones de la investigación de los sites, así como los datos son tratados según la metodología de análisis de redes adoptada. Por tanto, para este caso, la investigación de campo es complementaria, en el sentido de subsidiar el reconocimiento del tema específico del análisis de la red socio-técnica en cuestión.

\section{Consideraciones finales}

El concepto de red como instrumento analítico presenta la necesidad de nuevos abordajes metodológicos que dirijan las investigaciones sobre fenómenos sociotécnicos. Este artículo buscó contribuir a ese fin, específicamente cuando el medio técnico de intermediación es la Web. Primero, al trazar consideraciones epistemológicas y metodológicas con objeto de apoyar a la investigación en líneas conceptuales sólidas, y segundo, al delinear los pasos para el levantamiento de datos, su representación y análisis. El concepto de esfera-Web usado junto con el del ARS proporcionó una vía segura para la investigación de las redes sociotécnicas. La esfera-Web se mostró como un referencial analítico adecuado para evidenciar las influencias recíprocas entre las redes sociales y las estructuras de articulación en la Web. Siendo más específicos, la evaluación de los arreglos de gobernabilidad a partir de la estructura de los hiperenlaces proporcionó estímulos para el análisis de los casos estudiados. También los discernimientos obtenidos a través del análisis estructural fueron determinantes para una mejor comprensión de los objetos analizados. Cabe mencionar como perspectiva prometedora para el uso del ARS para el análisis de las redes socio-técnicas, así como su potencial para la integración de abordajes de investigación cualitativos y cuantitativos. Observamos que el abordaje cuantitativo permite evidenciar estructuras e interrelaciones que son de difícil aprehensión por parte de los abordajes cualitativos, fundamentados en la interpretación socio-histórica, al mismo tiempo en que sus resultados, si son considerados exclusivamente, pueden llevar a interpretaciones equivocadas.

Los procedimientos delineados fueron refinados a través de la aplicación de los estudios de caso. Las particularidades de las diferentes redes socio-técnicas en cuestión sugieren adecuaciones específicas en consonancia con los intereses específicos de cada investigación. En el caso de la red ambiental, por ejemplo, los factores de tamaño de la red, por un lado, y su fuerte inserción en el ámbito 
nacional y global, por otro, exigían líneas de corte en el sentido de una restricción de las instituciones con actuación local en Curitiba y relacionadas a la cuestión ambiental urbana. Los levantamientos corroboran el análisis de Manuel Castells (1999) que llamó la atención por el carácter transnacional del movimiento ambientalista, cuya capacidad de articulación global está estrechamente ligada al uso intensivo de las tecnologías de la información y comunicación. Mientras en lo referente a la esfera-Web de la política ambiental los movimientos y organizaciones de la sociedad civil organizada revelaron ser los agentes preponderantes, en la esfera-Web referente a la promoción de las tecnologías sociales se percibe una fuerte presencia del mercado, sea a través de empresas ligadas a la tecnología de la información o a las telecomunicaciones, sea a través de fundaciones $u$ organizaciones sociales mantenidas por empresas privadas o públicas.

Con relación al estudio sobre la red de protección social llama la atención que la red configurada a partir de los sites revela articulaciones y alianzas no siempre capturables en procedimientos de recolección de datos como la entrevista y la observación. Esto demuestra que el cotidiano de las actividades de estos agentes organizacionales puede encubrir, inclusive para algunos de sus representantes, intencionalidades, dinámicas, el histórico de afinidades temáticas e ideológicas, estándares de cooperación y potencializaciones mutuas entre los agentes envueltos, lo que apenas el documento site revela. En este sentido, queda claro que la red socio-técnica no es un "retrato" o una muestra de la red social de los agentes seleccionados, pero sí es una red (re)configurada por medio del propio procedimiento metodológico centrado en la Web, y por eso mismo revela perspectivas de análisis y comprensión tanto del tema, en este caso protección social en las ciudades, pero, también, de los propios agentes envueltos y su inserción en los lazos relacionales explicitados por medio de esta metodología.

Se resalta, sin embargo, que los límites impuestos por el objetivo de los estudios de caso, exigen posterior profundización en el entendimiento de sus interrelaciones con el desarrollo de la acción social. La complementación de los procedimientos de investigación se hace indispensable, por ejemplo, a través de la adopción de métodos cualitativos de interpretación histórica y el análisis del contenido de páginas Web, a través de métodos de análisis de discurso. Se torna así indispensable que los resultados obtenidos por el análisis de la esfera Web y del ARS puedan ser confrontados con estudios cualitativos complementarios sobre las formas de colaboración y de coordinación social, así como sobre los modos y las condiciones de cooperación interinstitucional y de la vida asociativa de los agentes 
envueltos en las redes estudiadas, inclusive para averiguar las interrelaciones e interdependencias existentes, los posibles conflictos o sinergias, entre las interacciones ocurrentes en el ámbito de la internet y en otras esferas de la vida social en las comunidades y municipios.

\section{Bibliografía}

Castells, M. (1999). A sociedade em rede. (A era da informação, sociedade e cultura: v1). São Paulo: Paz e Terra.

Duarte, F. (1998). Global e local no mundo contemporâneo. São Paulo: Moderna.

Emirbayer, M.; Goodwin, J. (1994). "Network analysis, culture, and the problem of agency". The American J ournal of Sociology, v.99, n.6, may, pp.1411-1454

Figueiredo, V. (2001). "A ciência da sociedade". 25으 Encontro Anual ANPOCS. disponível em< www.anpocs.org.br/ > acesso em 05/07/2006

Foot,K.A.; Schneider,S.M.; Dougherty,M; Xenos,M.; Larsen,E. (2003). “Analyzing Linking Practices: Candidate Sites in the 2002 US Electoral Web Sphere". JCMCJ ournal of Computer-Mediated Communication. V8 (4), July.

Frey, K. (2003). “Desenvolvimento sustentável local na emergente sociedade em rede: o potencial das novas tecnologias da informação e comunicação na formação de redes comunitárias". Revista de Sociologia e Política, no.21, Nov, p.165-185 (disponible en: http://www.scielo.br/pdf/rsocp/n21/a11n21.pdf; acceso en: 03/04/2006)

Frey, K. (2005). "ICT-enforced community networks for sustainable development and social inclusion". In: Albrechts, L.; Mandelbaum, Seymour, J. (eds.) The network society: a new context for planning. London \& New York: Routledge, p.183-196.

Frey, K.; Penna, M. C.; Czajkowski, S. (2005). "Governança urbana, redes de políticas públicas e sua análise". In: Anais do XXIX Encontro Anual da Associação Nacional de Pós-Graduação e Pesquisa em Ciências Sociais, São Paulo: ANPOCS, V.1, pp. 1-22.

Giddens, A. (1989). A constituição da sociedade, São Paulo: Martins Fontes.

Graham, S. and Marvin, S. (1996). "Rumo à cidade em tempo real. Desenvolvimento Urbano numa Sociedade Globalizada e Telemediática" in: Telecommunications and the City: Electronic Spaces, Urban Spaces. New York: Routledge (traducción disponible en: www.wisetel.com.br/espaco_de_futuros/vcidade.htm; acceso en: 07/06/2006)

Jansen, D. (2003). Einführung in die Netzwerkanalyse. Opladen: Leske + Budrich.

Lamb, R; Davidson, E. (2002). "Social Scientists: managing identity in sociotechnical networks". Proceedings of the Hawai's International Conference on System Sciences, January 7-10, Big Island, Hawaii; disponible en: http://lamb.cba.hawaii. edu/pubs/socialscientists. pdf (acceso: 04/02/2006) 
Loiola, E.; Moura, S. (1997). "Análise de redes: uma contribuição aos estudos organizacionais". In: Fisher, T. (org.). Gestão contemporânea, cidades estratégicas e organizações locais. Rio de Janeiro: FGV, p.53-68.

Martins, P. H. (2004). "As redes sociais, o sistema da dádiva e o paradoxo sociológico". In: Martins, P. H.; Fontes, B. (orgs.). Redes sociais e saúde: novas possibilidades teóricas. Recife: Ed. Univ. da UFPE, p.21-48

Mitra, A. (1999). Characteristics of the WWW text: tracing discursive strategies, J ournal of Computer Mediated Communication, vol(5)1, September.

Musso, P. (2004). "A filosofia da rede". In: Parente, A. (org.) Tramas da rede: novas dimensões filosóficas, estéticas e políticas da comunicação. Porto Alegre: Sulina

Neustadt, A.; Robinson, J.P.; Kestnbaum, M. (2002). "Doing social science research online". In: Wellman, B.; Haythornthwaite, C.A. (eds.). The Internet in everyday life. Malden/Oxford: Blackwell, p.186-211

Park, H.W.; Barnett, G.A; Nam, Y. (2002). "Interorganizational Hiperenlace Networks among Websites in South Korea. Networks and Communication Studies". Netcom, vol. 16, $\mathrm{n}^{\circ}$ 3-4. p. 155-174.

Parrochia, D. (1993). Philosophie dês réseaux. Paris: PUF.

Prado, G. (2003). Arte telemática: dos intercâmbios pontuais aos ambientes virtuais multiusuário. São Paulo: I taú Cultural.

Rothenberg, R. et al (1998). "Social network dynamics and HIV transmission". AIDS: Volume 12(12) 20 August, pp. 1529-1536

Santos, M. (2002). A natureza do espaço. Técnica e tempo. Razão e emoção. São Paulo: EdUSP.

Scherer-Warren, I. (2005). "Redes sociais: trajetórias e fronteiras". In: Dias, L. e Silveira, R. L. (org.). Redes, sociedades e territórios. Santa Cruz do Sul: Edunisc, p.29-50.

Schneider, S.M.; Foot,K.A. (2005). "Web Sphere Analysis: An Approach to Studying Online Action". In Hine, C. (ed.). Virtual methods: issues in social science research on the Internet. Berg Publishers, Oxford. Disponible en: www. sunyit. edu/ steve/ papers/schneider-foot-Websphereanalysis-0040303dist. pdf

Scott, J. (2000). Social Network Analysis: a Handbook - Second Edition, SAGE Publications: London.

Vilasante, T. R. (2002). Redes e alternativas: estratégias e estilos criativos na complexidade social. Petrópolis, RJ : Vozes.

Wasserman, S.; Faust, K. (1994). Social network analysis: methods and applications. Cambridge: Cambridge University Press. 